Homology, Homotopy and Applications, vol.20(1), 2018, pp.141-154

\title{
A HOMOTOPY DECOMPOSITION OF THE FIBRE OF THE SQUARING MAP ON $\Omega^{3} S^{17}$
}

\author{
STEVEN AMELOTTE
}

(communicated by Donald M. Davis)

\begin{abstract}
We use Richter's 2-primary proof of Gray's conjecture to give a homotopy decomposition of the fibre $\Omega^{3} S^{17}\{2\}$ of the $H$-space squaring map on the triple loop space of the 17-sphere. This induces a splitting of the mod-2 homotopy groups $\pi_{*}\left(S^{17} ; \mathbb{Z} / 2 \mathbb{Z}\right)$ in terms of the integral homotopy groups of the fibre of the double suspension $E^{2}: S^{2 n-1} \rightarrow \Omega^{2} S^{2 n+1}$ and refines a result of Cohen and Selick, who gave similar decompositions for $S^{5}$ and $S^{9}$. We relate these decompositions to various Whitehead products in the homotopy groups of mod-2 Moore spaces and Stiefel manifolds to show that the Whitehead square $\left[i_{2 n}, i_{2 n}\right]$ of the inclusion of the bottom cell of the Moore space $P^{2 n+1}(2)$ is divisible by 2 if and only if $2 n=2,4,8$ or 16 .
\end{abstract}

\section{Introduction}

For a based loop space $\Omega X$, let $\Omega X\{k\}$ denote the homotopy fibre of the $k$ th power map $k: \Omega X \rightarrow \Omega X$. In [14] and [15], Selick showed that after localizing at an odd prime $p$, there is a homotopy decomposition $\Omega^{2} S^{2 p+1}\{p\} \simeq \Omega^{2} S^{3}\langle 3\rangle \times W_{p}$, where $S^{3}\langle 3\rangle$ is the 3 -connected cover of $S^{3}$ and $W_{n}$ is the homotopy fibre of the double suspension $E^{2}: S^{2 n-1} \rightarrow \Omega^{2} S^{2 n+1}$. Since $\Omega^{2} S^{2 p+1}\{p\}$ is homotopy equivalent to the pointed mapping space $\operatorname{Map}_{*}\left(P^{3}(p), S^{2 p+1}\right)$ and the degree $p$ map on the Moore space $P^{3}(p)$ is nullhomotopic, an immediate consequence is that $p$ annihilates the $p$-torsion in $\pi_{*}\left(S^{3}\right)$ when $p$ is odd. In [16], Ravenel's solution to the odd primary Arf-Kervaire invariant problem [12] was used to show that, at least for $p \geqslant 5$, similar decompositions of $\Omega^{2} S^{2 n+1}\{p\}$ are not possible if $n \neq 1$ or $p$.

The 2-primary analogue of Selick's decomposition, namely that there is a 2-local homotopy equivalence $\Omega^{2} S^{5}\{2\} \simeq \Omega^{2} S^{3}\langle 3\rangle \times W_{2}$, was later proved by Cohen [4]. Similarly, since $\Omega^{2} S^{5}\{2\}$ is homotopy equivalent to $\operatorname{Map}_{*}\left(P^{3}(2), S^{5}\right)$ and the degree 4 map on $P^{3}(2) \simeq \Sigma \mathbb{R} P^{2}$ is nullhomotopic, this product decomposition gives a "geometric" proof of James' classical result that 4 annihilates the 2-torsion in $\pi_{*}\left(S^{3}\right)$. Unlike the odd primary case, however, for reasons related to the divisibility of the Whitehead square $\left[\iota_{2 n-1}, \iota_{2 n-1}\right] \in \pi_{4 n-3}\left(S^{2 n-1}\right)$, the fibre of the squaring map on $\Omega^{2} S^{2 n+1}$ admits nontrivial product decompositions for some other values of $n$.

Received July 20, 2017, revised August 31, 2017; published on January 24, 2018.

2010 Mathematics Subject Classification: 55P35, 55P10, 55Q15.

Key words and phrases: loop space decomposition, Moore space, Whitehead product.

Article available at http://dx.doi.org/10.4310/HHA.2018.v20.n1.a9

Copyright (c) 2018, International Press. Permission to copy for private use granted. 
First, in their investigation of the homology of spaces of maps from mod-2 Moore spaces to spheres, Campbell, Cohen, Peterson and Selick [1] found that if $2 n+1 \neq$ $3,5,9$ or 17 , then $\Omega^{2} S^{2 n+1}\{2\}$ is atomic and hence indecomposable. Following this, it was shown in [5] that after localization at the prime 2, there is a homotopy decomposition $\Omega^{2} S^{9}\{2\} \simeq B W_{2} \times W_{4}$ and $W_{4}$ is a retract of $\Omega^{3} S^{17}\{2\}$. Here $B W_{n}$ denotes the classifying space of $W_{n}$ first constructed by Gray [6]. Since $B W_{1}$ is known to be homotopy equivalent to $\Omega^{2} S^{3}\langle 3\rangle$, the pattern suggested by the decompositions of $\Omega^{2} S^{5}\{2\}$ and $\Omega^{2} S^{9}\{2\}$ led Cohen and Selick to conjecture that $\Omega^{2} S^{17}\{2\} \simeq B W_{4} \times W_{8}$. In this note we prove this is true after looping once. (This weaker statement was also conjectured in $[\mathbf{3}]$.)

Theorem 1.1. There is a 2-local homotopy equivalence $\Omega^{3} S^{17}\{2\} \simeq W_{4} \times \Omega W_{8}$.

In addition to the exponent results mentioned above, decompositions of $\Omega^{m} S^{2 n+1}\{p\}$ also give decompositions of homotopy groups of spheres with $\mathbb{Z} / p \mathbb{Z}$ coefficients. Recall that the mod- $p$ homotopy groups of $X$ are defined by $\pi_{k}(X ; \mathbb{Z} / p \mathbb{Z})=$ $\left[P^{k}(p), X\right]$.

Corollary 1.2. $\pi_{k}\left(S^{17} ; \mathbb{Z} / 2 \mathbb{Z}\right) \cong \pi_{k-4}\left(W_{4}\right) \oplus \pi_{k-3}\left(W_{8}\right)$ for all $k \geqslant 4$.

In Section 3 we relate the problem of decomposing $\Omega^{2} S^{2 n+1}\{2\}$ to a problem considered by Mukai and Skopenkov in [11] of computing a certain summand in a homotopy group of the mod-2 Moore space $P^{2 n+1}(2)$ - more specifically, the problem of determining when the Whitehead square $\left[i_{2 n}, i_{2 n}\right] \in \pi_{4 n-1}\left(P^{2 n+1}(2)\right)$ of the inclusion of the bottom cell $i_{2 n}: S^{2 n} \rightarrow P^{2 n+1}(2)$ is divisible by 2 . The indecomposability result for $\Omega^{2} S^{2 n+1}\{2\}$ in [1] (see also [2]) was proved by showing that for $n>1$ the existence of a spherical homology class in $H_{4 n-3}\left(\Omega^{2} S^{2 n+1}\{2\}\right)$ imposed by a nontrivial product decomposition implies the existence of an element $\theta \in \pi_{2 n-2}^{S}$ of Kervaire invariant one such that $\theta \eta$ is divisible by 2 , where $\eta$ is the generator of the stable 1 -stem $\pi_{1}^{S}$. Such elements are known to exist only for $2 n=4,8$ or 16 . We show that the divisibility of the Whitehead square $\left[i_{2 n}, i_{2 n}\right]$ similarly implies the existence of such Kervaire invariant elements to obtain the following.

Theorem 1.3. The Whitehead square $\left[i_{2 n}, i_{2 n}\right] \in \pi_{4 n-1}\left(P^{2 n+1}(2)\right)$ is divisible by 2 if and only if $2 n=2,4,8$ or 16 .

This will follow from a preliminary result (Proposition 3.1) equating the divisibility of $\left[i_{2 n}, i_{2 n}\right]$ with the vanishing of a Whitehead product in the mod-2 homotopy of the Stiefel manifold $V_{2 n+1,2}$, i.e., the unit tangent bundle over $S^{2 n}$. It is shown in [17] that there do not exist maps $S^{2 n-1} \times P^{2 n}(2) \rightarrow V_{2 n+1,2}$ extending the inclusions of the bottom cell $S^{2 n-1}$ and bottom Moore space $P^{2 n}(2)$ if $2 n \neq 2,4,8$ or 16 . When $2 n=2,4$ or 8 , the Whitehead product obstructing an extension is known to vanish for reasons related to Hopf invariant one, leaving only the boundary case $2 n=16$ unresolved. We find that the Whitehead product is also trivial in this case.

\section{Acknowledgments}

The author would like to thank his advisor, Paul Selick, for his guidance and support, Stephen Theriault for reading a draft of this paper, and the referee for their feedback. The author was supported by an OGS scholarship during the preparation of this work. 


\section{The decomposition of $\Omega^{3} S^{17}\{2\}$}

The proof of Theorem 1.1 will make use of the 2-primary version of Richter's proof of Gray's conjecture, so we begin by reviewing this conjecture and spelling out some of its consequences. In his construction of a classifying space of the fibre $W_{n}$ of the double suspension, Gray [6] introduced two $p$-local homotopy fibrations

$$
\begin{aligned}
& S^{2 n-1} \stackrel{E^{2}}{\longrightarrow} \Omega^{2} S^{2 n+1} \stackrel{\nu}{\longrightarrow} B W_{n}, \\
& B W_{n} \stackrel{j}{\longrightarrow} \Omega^{2} S^{2 n p+1} \stackrel{\phi}{\longrightarrow} S^{2 n p-1},
\end{aligned}
$$

with the property that $j \circ \nu \simeq \Omega H$, where $H: \Omega S^{2 n+1} \rightarrow \Omega S^{2 n p+1}$ is the $p$ th JamesHopf invariant. In addition, Gray showed that the composite $B W_{n} \stackrel{j}{\rightarrow} \Omega^{2} S^{2 n p+1} \stackrel{p}{\rightarrow}$ $\Omega^{2} S^{2 n p+1}$ is nullhomotopic and conjectured that the composite $\Omega^{2} S^{2 n p+1} \stackrel{\phi}{\rightarrow}$ $S^{2 n p-1} \stackrel{E^{2}}{\longrightarrow} \Omega^{2} S^{2 n p+1}$ is homotopic to the $p$ th power map on $\Omega^{2} S^{2 n p+1}$. This was recently proved by Richter in $[\mathbf{1 3}]$.

Theorem 2.1 ([13]). For any prime $p$, there is a homotopy fibration

$$
B W_{n} \stackrel{j}{\longrightarrow} \Omega^{2} S^{2 n p+1} \stackrel{\phi_{n}}{\longrightarrow} S^{2 n p-1}
$$

such that $E^{2} \circ \phi_{n} \simeq p$.

For odd primes, it was shown in [21] that there is a homotopy fibration $\Omega W_{n p} \rightarrow$ $B W_{n} \rightarrow \Omega^{2} S^{2 n p+1}\{p\}$ based on the fact that a lift $\bar{S}: B W_{n} \rightarrow \Omega^{2} S^{2 n p+1}\{p\}$ of $j$ can be chosen to be an $H$-map when $p$ is odd. One consequence of Theorem 2.1 is that this homotopy fibration exists for all primes and can be extended one step to the right by a map $\Omega^{2} S^{2 n p+1}\{p\} \rightarrow W_{n p}$.

Lemma 2.2. For any prime $p$, there is a homotopy fibration

$$
B W_{n} \longrightarrow \Omega^{2} S^{2 n p+1}\{p\} \longrightarrow W_{n p} .
$$

Proof. The homotopy pullback of $\phi_{n}$ and the fibre inclusion $W_{n p} \rightarrow S^{2 n p-1}$ of the double suspension defines a map $\Omega^{2} S^{2 n p+1}\{p\} \rightarrow W_{n p}$ with homotopy fibre $B W_{n}$, which can be seen by comparing fibres in the homotopy pullback diagram

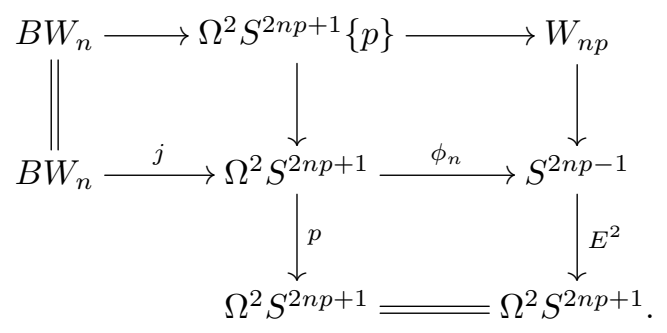

Looping once, we obtain a homotopy fibration

$$
W_{n} \longrightarrow \Omega^{3} S^{2 n p+1}\{p\} \longrightarrow \Omega W_{n p}
$$

which we will show is split when $p=2$ and $n=4$. We now fix $p=2$ and localize all spaces and maps at the prime 2 . Homology will be taken with mod- 2 coefficients unless otherwise stated. 
The next lemma describes a factorization of the looped second James-Hopf invariant, an odd primary version of which appears in [21]. By a well-known result due to Barratt, $\Omega H: \Omega^{2} S^{2 n+1} \rightarrow \Omega^{2} S^{4 n+1}$ has order 2 in the group $\left[\Omega^{2} S^{2 n+1}, \Omega^{2} S^{4 n+1}\right]$ and hence lifts to a map $\Omega^{2} S^{2 n+1} \rightarrow \Omega^{2} S^{4 n+1}\{2\}$. Improving on this, a feature of Richter's construction of the map $\phi_{n}$ is that the composite $\Omega^{2} S^{2 n+1} \stackrel{\Omega H}{\longrightarrow} \Omega^{2} S^{4 n+1} \stackrel{\phi_{n}}{\longrightarrow} S^{4 n-1}$ is nullhomotopic [13, Lemma 4.2]. This recovers Gray's fibration $S^{2 n-1} \stackrel{E^{2}}{\rightarrow} \Omega^{2} S^{2 n+1} \stackrel{\nu}{\rightarrow}$ $B W_{n}$ and the relation $j \circ \nu \simeq \Omega H$ since there then exists a lift $\nu: \Omega^{2} S^{2 n+1} \rightarrow B W_{n}$ making the diagram

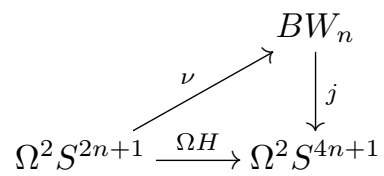

commute up to homotopy. Since $j$ factors through $\Omega^{2} S^{4 n+1}\{2\}$, by composing the lift $\nu$ with the map $B W_{n} \rightarrow \Omega^{2} S^{4 n+1}\{2\}$ we obtain a choice of lift $S: \Omega^{2} S^{2 n+1} \rightarrow$ $\Omega^{2} S^{4 n+1}\{2\}$ of the looped James-Hopf invariant. Hence we have the following consequence of Richter's theorem.

Lemma 2.3. There is a homotopy commutative diagram

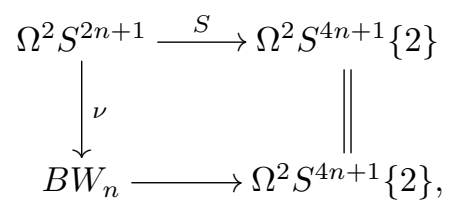

where $S$ is a lift of the looped second James-Hopf invariant $\Omega H: \Omega^{2} S^{2 n+1} \rightarrow \Omega^{2} S^{4 n+1}$ and the map $B W_{n} \rightarrow \Omega^{2} S^{4 n+1}\{2\}$ has homotopy fibre $\Omega W_{2 n}$.

The following homological result was proved in $[\mathbf{1}]$ and used to obtain the homotopy decompositions of [4] and [5].

Lemma 2.4 ([1] $)$. Let $n \geqslant 2$ and let $f: X \rightarrow \Omega^{2} S^{2 n+1}\{2\}$ be a map which induces an isomorphism on the module of primitives in degrees $2 n-2$ and $4 n-3$. If the mod-2 homology of $X$ is isomorphic to that of $\Omega^{2} S^{2 n+1}\{2\}$ as a coalgebra over the Steenrod algebra, then $f$ is a homology isomorphism.

Theorem 2.5. There is a homotopy equivalence $\Omega^{3} S^{17}\{2\} \simeq W_{4} \times \Omega W_{8}$.

Proof. Let $\tau_{n}$ denote the map $B W_{n} \rightarrow \Omega^{2} S^{4 n+1}\{2\}$ appearing in Lemma 2.2. By (1), $\tau_{n}$ is a lift of $j$, implying that $\tau_{n}$ is nonzero in $H_{4 n-2}()$ by naturality of the Bockstein since $j$ is nonzero in $H_{4 n-1}()$. We can therefore use the maps $\tau_{n}$ in place of the (potentially different) maps $\sigma_{n}$ used in [5] to obtain product decompositions of $\Omega^{2} S^{4 n+1}\{2\}$ for $n=1$ and 2, the advantage being that $\tau_{n}$ has fibre $\Omega W_{2 n}$. Explicitly, for $n=2$ this is done as follows. By [5, Corollary 2.1], there exists a map $g: \Omega^{3} S^{17}\{2\} \rightarrow \Omega^{2} S^{9}\{2\}$ which is nonzero in $H_{13}()$. Letting $\mu$ denote the loop multiplication on $\Omega^{2} S^{9}\{2\}$, it follows that the composite

$$
\psi: B W_{2} \times W_{4} \stackrel{\tau_{2} \times\left(g \circ \Omega \tau_{4}\right)}{\longrightarrow} \Omega^{2} S^{9}\{2\} \times \Omega^{2} S^{9}\{2\} \stackrel{\mu}{\longrightarrow} \Omega^{2} S^{9}\{2\}
$$

induces an isomorphism on the module of primitives in degrees 6 and 13. Since 
$H_{*}\left(B W_{2} \times W_{4}\right)$ and $H_{*}\left(\Omega^{2} S^{9}\{2\}\right)$ are isomorphic as coalgebras over the Steenrod algebra, the map above is a homology isomorphism by Lemma 2.4 and hence a homotopy equivalence.

Now the map $\Omega \tau_{4}$ fits in the homotopy fibration

$$
W_{4} \stackrel{\Omega \tau_{4}}{\longrightarrow} \Omega^{3} S^{17}\{2\} \longrightarrow \Omega W_{8}
$$

and has a left homotopy inverse given by $\pi_{2} \circ \psi^{-1} \circ g$ where $\psi^{-1}$ is a homotopy inverse of $\psi$ and $\pi_{2}: B W_{2} \times W_{4} \rightarrow W_{4}$ is the projection onto the second factor. (Alternatively, composing $g: \Omega^{3} S^{17}\{2\} \rightarrow \Omega^{2} S^{9}\{2\}$ with the map $\Omega^{2} S^{9}\{2\} \rightarrow W_{4}$ of Lemma 2.2 yields a left homotopy inverse of $\Omega \tau_{4}$.) It follows that the homotopy fibration above is fibre homotopy equivalent to the trivial fibration $W_{4} \times \Omega W_{8} \rightarrow \Omega W_{8}$.

Corollary 2.6. $\pi_{k}\left(S^{17} ; \mathbb{Z} / 2 \mathbb{Z}\right) \cong \pi_{k-4}\left(W_{4}\right) \oplus \pi_{k-3}\left(W_{8}\right)$ for all $k \geqslant 4$.

One consequence of the splitting of the fibration $W_{n} \rightarrow \Omega^{3} S^{4 n+1}\{p\} \rightarrow \Omega W_{2 n}$ when $n \in\{1,2,4\}$ is a corresponding homotopy decomposition of the fibre of the map $S$ appearing in Lemma 2.3. As in [18], we define the space $Y$ and the map $t$ by the homotopy fibration

$$
Y \stackrel{t}{\longrightarrow} \Omega^{2} S^{2 n+1} \stackrel{S}{\longrightarrow} \Omega^{2} S^{4 n+1}\{2\} .
$$

This space and its odd primary analogue play a central role in the construction of Anick's fibration in $[\mathbf{1 8}, \mathbf{2 1}]$ and the alternative proof given in $[\mathbf{2 0}]$ of Cohen, Moore and Neisendorfer's determination of the odd primary homotopy exponent of spheres. Unlike at odd primes, the lift $S$ of $\Omega H$ cannot be chosen to be an $H$-map. Nevertheless, the corollary below shows that its fibre has the structure of an $H$-space in cases of Hopf invariant one.

Corollary 2.7. There is a homotopy fibration $S^{2 n-1} \stackrel{f}{\rightarrow} Y \stackrel{g}{\rightarrow} \Omega W_{2 n}$ with the property that the composite $S^{2 n-1} \stackrel{f}{\rightarrow} Y \stackrel{t}{\rightarrow} \Omega^{2} S^{2 n+1}$ is homotopic to the double suspension $E^{2}$. Moreover, if $n=1,2$ or 4 then the fibration splits, giving a homotopy equivalence

$$
Y \simeq S^{2 n-1} \times \Omega W_{2 n}
$$

Proof. By Lemma 2.3, the homotopy fibration defining $Y$ fits in a homotopy pullback diagram

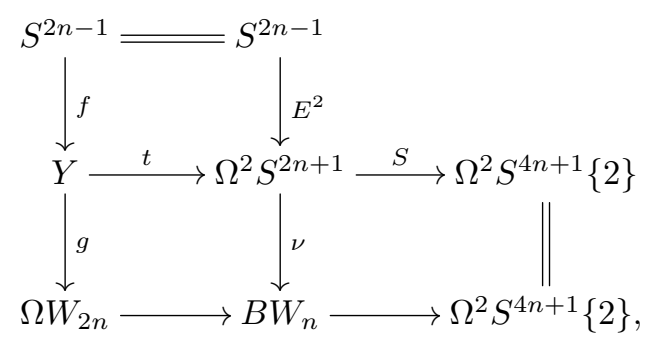

which proves the first statement. Note that when $n=1,2$ or 4 , the map $\Omega W_{2 n} \rightarrow$ $B W_{n}$ is nullhomotopic by Theorem 1.1, hence $t$ lifts through the double suspension. Since any choice of a lift $Y \rightarrow S^{2 n-1}$ is degree one in $H_{2 n-1}()$, it also serves as a left homotopy inverse of $f$, which implies the asserted splitting. 
Remark 2.8. The first part of Corollary 2.7 and an odd primary version are proved by different means in [18] and [20], respectively (see Remark 6.2 of [18]). At odd primes, there is an analogous splitting for $n=1$ :

$$
Y \simeq S^{1} \times \Omega W_{p} \simeq S^{1} \times \Omega^{3} T^{2 p^{2}+1}(p),
$$

where $T^{2 p^{2}+1}(p)$ is Anick's space (see [19]).

\section{Relations to Whitehead products in Moore spaces and Stiefel manifolds}

The special homotopy decompositions of $\Omega^{3} S^{2 n+1}\{2\}$ discussed in the previous section are made possible by the existence of special elements in the stable homotopy groups of spheres, namely elements of Arf-Kervaire invariant one $\theta \in \pi_{2 n-2}^{S}$ such that $\theta \eta$ is divisible by 2 . In this section, we give several reformulations of the existence of such elements in terms of mod-2 Moore spaces and Stiefel manifolds.

Let $i_{n-1}: S^{n-1} \rightarrow P^{n}(2)$ be the inclusion of the bottom cell and let $j_{n}: P^{n}(2) \rightarrow$ $P^{n}(2)$ be the identity map. Similarly, let $i_{2 n-1}^{\prime}: S^{2 n-1} \rightarrow V_{2 n+1,2}$ and $j_{2 n}^{\prime}: P^{2 n}(2) \rightarrow$ $V_{2 n+1,2}$ denote the inclusions of the bottom cell and bottom Moore space, respectively. ${ }^{1}$

Proposition 3.1. The Whitehead product $\left[i_{2 n-1}^{\prime}, j_{2 n}^{\prime}\right] \in \pi_{4 n-2}\left(V_{2 n+1,2} ; \mathbb{Z} / 2 \mathbb{Z}\right)$ is trivial if and only if the Whitehead square $\left[i_{2 n}, i_{2 n}\right] \in \pi_{4 n-1}\left(P^{2 n+1}(2)\right)$ is divisible by 2 .

Proof. Let $\lambda: S^{4 n-2} \rightarrow P^{2 n}(2)$ denote the attaching map of the top cell in $V_{2 n+1,2} \simeq$ $P^{2 n}(2) \cup_{\lambda} e^{4 n-1}$ and note that $\left[i_{2 n-1}^{\prime}, j_{2 n}^{\prime}\right]=j_{2 n}^{\prime} \circ\left[i_{2 n-1}, j_{2 n}\right]$ by naturality of the Whitehead product. The map $\left[i_{2 n-1}, j_{2 n}\right]: P^{4 n-2}(2) \rightarrow P^{2 n}(2)$ is essential since its adjoint is a Samelson product with nontrivial Hurewicz image $[u, v] \in$ $H_{4 n-3}\left(\Omega P^{2 n}(2)\right)$, where $H_{*}\left(\Omega P^{2 n}(2)\right)$ is isomorphic as an algebra to the tensor algebra $T(u, v)$ with $|u|=2 n-2$ and $|v|=2 n-1$ by the Bott-Samelson theorem. Since the homotopy fibre of the inclusion $j_{2 n}^{\prime}: P^{2 n}(2) \rightarrow V_{2 n+1,2}$ has $(4 n-2)$-skeleton $S^{4 n-2}$ which maps into $P^{2 n}(2)$ by the attaching map $\lambda$, it follows that $\left[i_{2 n-1}^{\prime}, j_{2 n}^{\prime}\right]$ is trivial if and only if $\left[i_{2 n-1}, j_{2 n}\right]$ is homotopic to the composite

$$
P^{4 n-2}(2) \stackrel{q}{\longrightarrow} S^{4 n-2} \stackrel{\lambda}{\longrightarrow} P^{2 n}(2),
$$

where $q$ is the pinch map.

To ease notation let $P^{n}$ denote the mod-2 Moore space $P^{n}(2)$ and consider the morphism of $E H P$ sequences

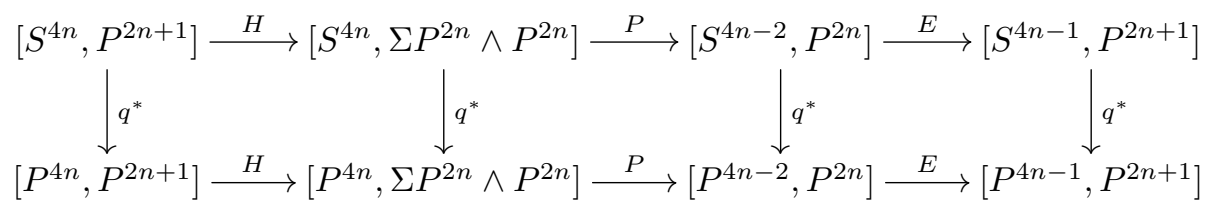

induced by the pinch map. A homology calculation shows that the $(4 n)$-skeleton of $\Sigma P^{2 n} \wedge P^{2 n}$ is homotopy equivalent to $P^{4 n} \vee S^{4 n}$. Let $k_{1}: P^{4 n} \rightarrow \Sigma P^{2 n} \wedge P^{2 n}$ and

\footnotetext{
${ }^{1}$ Note that we index these maps by the dimension of their source rather than their target, so the element of $\pi_{4 n-1}\left(P^{2 n+1}(2)\right)$ we call $\left[i_{2 n}, i_{2 n}\right]$ is called $\left[i_{2 n+1}, i_{2 n+1}\right]$ in [11].
} 
$k_{2}: S^{4 n} \rightarrow \Sigma P^{2 n} \wedge P^{2 n}$ be the composites

$$
P^{4 n} \hookrightarrow P^{4 n} \vee S^{4 n} \simeq \operatorname{sk}_{4 n}\left(\Sigma P^{2 n} \wedge P^{2 n}\right) \hookrightarrow \Sigma P^{2 n} \wedge P^{2 n}
$$

and

$$
S^{4 n} \hookrightarrow P^{4 n} \vee S^{4 n} \simeq \operatorname{sk}_{4 n}\left(\Sigma P^{2 n} \wedge P^{2 n}\right) \hookrightarrow \Sigma P^{2 n} \wedge P^{2 n}
$$

defined by the left and right wedge summand inclusions, respectively. Then we have that $\pi_{4 n}\left(\Sigma P^{2 n} \wedge P^{2 n}\right)=\mathbb{Z} / 4 \mathbb{Z}\left\{k_{2}\right\}$ and $P\left(k_{2}\right)= \pm 2 \lambda$ by [9, Lemma 12]. It follows from the universal coefficient exact sequence

$$
\begin{aligned}
0 \longrightarrow \pi_{4 n}\left(\Sigma P^{2 n} \wedge P^{2 n}\right) \otimes \mathbb{Z} / 2 \mathbb{Z} \longrightarrow \pi_{4 n}\left(\Sigma P^{2 n} \wedge P^{2 n} ; \mathbb{Z} / 2 \mathbb{Z}\right) & \\
\longrightarrow & \operatorname{Tor}\left(\pi_{4 n-1}\left(\Sigma P^{2 n} \wedge P^{2 n}\right), \mathbb{Z} / 2 \mathbb{Z}\right) \longrightarrow 0
\end{aligned}
$$

that

$$
\begin{aligned}
\pi_{4 n}\left(\Sigma P^{2 n} \wedge P^{2 n} ; \mathbb{Z} / 2 \mathbb{Z}\right) & =\left[P^{4 n}, \Sigma P^{2 n} \wedge P^{2 n}\right] \\
& =\mathbb{Z} / 2 \mathbb{Z}\left\{k_{1}\right\} \oplus \mathbb{Z} / 2 \mathbb{Z}\left\{k_{2} \circ q\right\}
\end{aligned}
$$

and that the generator $k_{2} \circ q$ is in the kernel of $P$ since $P\left(k_{2}\right)= \pm 2 \lambda$ implies

$$
P\left(k_{2} \circ q\right)=P\left(q^{*}\left(k_{2}\right)\right)=q^{*}\left(P\left(k_{2}\right)\right)= \pm \lambda \circ 2 \circ q=0
$$

by the commutativity of the above diagram and the fact that $q: P^{4 n-2} \rightarrow S^{4 n-2}$ and 2: $S^{4 n-2} \rightarrow S^{4 n-2}$ are consecutive maps in a cofibration sequence. Therefore $\left[i_{2 n-1}, j_{2 n}\right]=P\left(k_{1}\right)$ since the suspension of a Whitehead product is trivial. On the other hand, $\Sigma \lambda$ is homotopic to the composite $S^{4 n-1} \stackrel{\left[\iota_{2 n}, \iota_{2 n}\right]}{\longrightarrow} S^{2 n} \stackrel{i_{2 n}}{\longrightarrow} P^{2 n+1}$ by [9], which implies $E(\lambda \circ q)=i_{2 n} \circ\left[\iota_{2 n}, \iota_{2 n}\right] \circ q=\left[i_{2 n}, i_{2 n}\right] \circ q$ is trivial in $\left[P^{4 n-1}, P^{2 n+1}\right]$ precisely when $\left[i_{2 n}, i_{2 n}\right]$ is divisible by 2 . Hence $\left[i_{2 n}, i_{2 n}\right]$ is divisible by 2 if and only if $\lambda \circ q=P\left(k_{1}\right)=\left[i_{2 n-1}, j_{2 n}\right] \in\left[P^{4 n-2}, P^{2 n}\right]$, and the proposition follows.

We use Proposition 3.1 in two ways. First, since the calculation of $\pi_{31}\left(P^{17}(2)\right)$ in $[10]$ shows that $\left[i_{16}, i_{16}\right]=2 \widetilde{\sigma}_{16}^{2}$ for a suitable choice of representative $\widetilde{\sigma}_{16}^{2}$ of the Toda bracket $\left\{\sigma_{16}^{2}, 2 \iota_{16}, i_{16}\right\}$, it follows that the Whitehead product $\left[i_{15}^{\prime}, j_{16}^{\prime}\right]: P^{30}(2) \rightarrow$ $V_{17,2}$ is nullhomotopic and hence there exists a map $S^{15} \times P^{16}(2) \rightarrow V_{17,2}$ extending the wedge of skeletal inclusions $S^{15} \vee P^{16}(2) \rightarrow V_{17,2}$. This resolves the only case left unsettled by Theorem 3.2 of $[\mathbf{1 7}]$.

In the other direction, note that such maps $S^{2 n-1} \times P^{2 n}(2) \rightarrow V_{2 n+1,2}$ restrict to maps $S^{2 n-1} \times S^{2 n-1} \rightarrow V_{2 n+1,2}$ which exist only in cases of Kervaire invariant one by [22, Proposition 2.27], so Proposition 3.1 shows that when $2 n \neq 2^{k}$ for some $k \geqslant 1$ the Whitehead square $\left[i_{2 n}, i_{2 n}\right]$ cannot be divisible by 2 for the same reasons that the Whitehead square $\left[\iota_{2 n-1}, \iota_{2 n-1}\right] \in \pi_{4 n-3}\left(S^{2 n-1}\right)$ cannot be divisible by 2 . Moreover, since maps $S^{2 n-1} \times P^{2 n}(2) \rightarrow V_{2 n+1,2}$ extending the inclusions of $S^{2 n-1}$ and $P^{2 n}(2)$ are shown not to exist for $2 n>16$ in [17], Proposition 3.1 implies that the Whitehead square $\left[i_{2 n}, i_{2 n}\right]$ is divisible by 2 if and only if $2 n=2,4,8$ or 16 . In all other cases it generates a $\mathbb{Z} / 2 \mathbb{Z}$ summand in $\pi_{4 n-1}\left(P^{2 n+1}(2)\right)$. This improves on the main theorem of $[\mathbf{1 1}]$ which shows by other means that $\left[i_{2 n}, i_{2 n}\right]$ is not divisible by 2 when $2 n$ is not a power of 2 .

These results are summarized in Theorem 3.3 below. First we recall the following well-known equivalent formulations of the Kervaire invariant problem. 
Theorem 3.2 ([2, 22]). The following are equivalent:

(a) The Whitehead square $\left[\iota_{2 n-1}, \iota_{2 n-1}\right] \in \pi_{4 n-3}\left(S^{2 n-1}\right)$ is divisible by 2 ;

(b) There is a map $P^{4 n-2}(2) \rightarrow \Omega S^{2 n}$ which is nonzero in homology;

(c) There exists a space $X$ with mod-2 cohomology $\widetilde{H}^{i}(X) \cong \mathbb{Z} / 2 \mathbb{Z}$ for $i=2 n$, $4 n-1$ and $4 n$, and zero otherwise, with $S q^{2 n}: H^{2 n}(X) \rightarrow H^{4 n}(X)$ and $S q^{1}: H^{4 n-1}(X) \rightarrow H^{4 n}(X)$ isomorphisms;

(d) There exists a map $f: S^{2 n-1} \times S^{2 n-1} \rightarrow V_{2 n+1,2}$ such that $\left.f\right|_{S^{2 n-1} \times *}=$ $\left.f\right|_{* \times S^{2 n-1}}$ is the inclusion of the bottom cell;

(e) $n=1$ or there exists an element $\theta \in \pi_{2 n-2}^{S}$ of Kervaire invariant one.

The above conditions hold for $2 n=2,4,8,16,32$ and 64 , and the recent solution to the Kervaire invariant problem by Hill, Hopkins and Ravenel [8] implies that, with the possible exception of $2 n=128$, these are the only values for which the conditions hold. Mimicking the reformulations above we obtain the following.

Theorem 3.3. The following are equivalent:

(a) The Whitehead square $\left[i_{2 n}, i_{2 n}\right] \in \pi_{4 n-1}\left(P^{2 n+1}(2)\right)$ is divisible by 2 ;

(b) There is a map $P^{4 n}(2) \rightarrow \Omega P^{2 n+2}(2)$ which is nonzero in homology;

(c) There exists a space $X$ with mod-2 cohomology $\widetilde{H}^{i}(X) \cong \mathbb{Z} / 2 \mathbb{Z}$ for $i=2 n+1$, $2 n+2,4 n+1,4 n+2$ and zero otherwise with $S q^{2 n}: H^{2 n+1}(X) \rightarrow H^{4 n+1}(X)$, $S q^{1}: H^{2 n+1}(X) \rightarrow H^{2 n+2}(X)$ and $S q^{1}: H^{4 n+1}(X) \rightarrow H^{4 n+2}(X)$ isomorphisms;

(d) There exists a map $f: S^{2 n-1} \times P^{2 n}(2) \rightarrow V_{2 n+1,2}$ such that $\left.f\right|_{S^{2 n-1} \times *}$ and $\left.f\right|_{* \times P^{2 n}(2)}$ are the skeletal inclusions of $S^{2 n-1}$ and $P^{2 n}(2)$, respectively;

(e) $n=1$ or there exists an element $\theta \in \pi_{2 n-2}^{S}$ of Kervaire invariant one such that $\theta \eta$ is divisible by 2 ;

(f) $2 n=2,4,8$ or 16 .

Proof. (a) is equivalent to (b): In the $n=1$ case, $\left[\iota_{2}, \iota_{2}\right]=2 \eta_{2}$ implies $\left[i_{2}, i_{2}\right]=0$, and since $\eta_{3} \in \pi_{4}\left(S^{3}\right)$ has order 2 its adjoint $\widetilde{\eta}_{3}: S^{3} \rightarrow \Omega S^{3}$ extends to a map $P^{4}(2) \rightarrow$ $\Omega S^{3}$. If this map desuspended, then $\widetilde{\eta}_{3}$ would be homotopic to a composite $S^{3} \rightarrow$ $P^{4}(2) \rightarrow S^{2} \stackrel{E}{\rightarrow} \Omega S^{3}$, a contradiction since $\pi_{3}\left(S^{2}\right) \cong \mathbb{Z}$ implies that any map $S^{3} \rightarrow$ $S^{2}$ that factors through $P^{4}(2)$ is nullhomotopic. Hence the map $P^{4}(2) \rightarrow \Omega S^{3}$ has nontrivial Hopf invariant in $\left[P^{4}(2), \Omega S^{5}\right]$ from which it follows that $P^{4}(2) \rightarrow \Omega S^{3}$ is nonzero in $H_{4}()$. Composing with the inclusion $\Omega S^{3} \rightarrow \Omega P^{4}(2)$ gives a map $P^{4}(2) \rightarrow$ $\Omega P^{4}(2)$ which is nonzero in $H_{4}()$.

Now suppose $n>1$ and $\left[i_{2 n}, i_{2 n}\right]=2 \alpha$ for some $\alpha \in \pi_{4 n-1}\left(P^{2 n+1}(2)\right)$. Then $\Sigma \alpha$ has order 2 so there is an extension $P^{4 n+1}(2) \rightarrow P^{2 n+2}(2)$ whose adjoint $f: P^{4 n}(2) \rightarrow$ $\Omega P^{2 n+2}(2)$ satisfies $\left.f\right|_{S^{4 n-1}}=E \circ \alpha$. Since $\Omega \Sigma\left(P^{2 n+1}(2) \wedge P^{2 n+1}(2)\right)$ has $4 n$-skeleton $S^{4 n}$, to show that $f_{*}$ is nonzero on $H_{4 n}\left(P^{4 n}(2)\right)$ it suffices to show that $H_{2} \circ f$ is nontrivial in $\left[P^{4 n}(2), \Omega \Sigma\left(P^{2 n+1}(2) \wedge P^{2 n+1}(2)\right)\right]$ where $H_{2}: \Omega P^{2 n+2}(2) \rightarrow \Omega \Sigma\left(P^{2 n+1}(2)\right.$ $\left.\wedge P^{2 n+1}(2)\right)$ is the second James-Hopf invariant. If $H_{2} \circ f$ is nullhomotopic, then 
there is a map $g: P^{4 n}(2) \rightarrow P^{2 n+1}(2)$ making the diagram

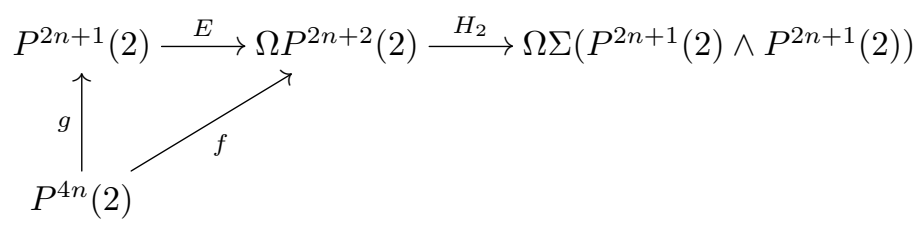

commute. But then $\alpha-\left.g\right|_{S^{4 n-1}}$ is in the kernel of $E_{*}: \pi_{4 n-1}\left(P^{2 n+1}(2)\right) \rightarrow$ $\pi_{4 n}\left(P^{2 n+2}(2)\right)$ which is generated by $\left[i_{2 n}, i_{2 n}\right]$, so $\alpha-\left.g\right|_{S^{4 n-1}}$ is a multiple of $\left[i_{2 n}, i_{2 n}\right]$. Since $\left[i_{2 n}, i_{2 n}\right]$ has order 2 and clearly $\left.2 g\right|_{S^{4 n-1}}=0$, it follows that $\left[i_{2 n}, i_{2 n}\right]=2 \alpha=0$, a contradiction. Therefore $f_{*}$ is nonzero on $H_{4 n}\left(P^{4 n}(2)\right)$.

Conversely, assume $n>1$ and $f: P^{4 n}(2) \rightarrow \Omega P^{2 n+2}(2)$ is nonzero in $H_{4 n}()$. Since the restriction $\left.f\right|_{S^{4 n-1}}$ lifts through the $(4 n-1)$-skeleton of $\Omega P^{2 n+2}(2)$, there is a homotopy commutative diagram

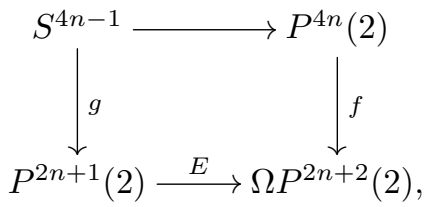

for some map $g: S^{4 n-1} \rightarrow P^{2 n+1}(2)$. Since $E \circ 2 g$ is nullhomotopic, $2 g$ is a multiple of $\left[i_{2 n}, i_{2 n}\right]$. But if $2 g=0$, then $g$ admits an extension $e: P^{4 n}(2) \rightarrow P^{2 n+1}(2)$ and it follows that $f-E \circ e$ factors through the pinch map $q: P^{4 n}(2) \rightarrow S^{4 n}$. This makes the Pontrjagin square $u^{2} \in H_{4 n}\left(\Omega P^{2 n+2}(2)\right)$ a spherical homology class, and this is a contradiction which can be seen as follows. If $u^{2}$ is spherical, then the $4 n$-skeleton of $\Omega P^{2 n+2}(2)$ is homotopy equivalent to $P^{2 n+1}(2) \vee S^{4 n}$. On the other hand, it is easy to see that the attaching map of the $4 n$-cell in $\Omega P^{2 n+2}(2)$ is given by the Whitehead square $\left[i_{2 n}, i_{2 n}\right]$ which is nontrivial as $n>1$, whence $P^{2 n+1} \cup_{\left[i_{2 n}, i_{2 n}\right]} e^{4 n} \not P^{2 n+1}(2) \vee$ $S^{4 n}$.

(a) is equivalent to (d): Since the Whitehead product $\left[i_{2 n-1}^{\prime}, j_{2 n}^{\prime}\right] \in \pi_{4 n-2}\left(V_{2 n+1,2}\right.$; $\mathbb{Z} / 2 \mathbb{Z})$ is the obstruction to extending $i_{2 n-1}^{\prime} \vee j_{2 n}^{\prime}: S^{2 n-1} \vee P^{2 n}(2) \rightarrow V_{2 n+1,2}$ to $S^{2 n-1} \times P^{2 n}(2)$, this follows immediately from Proposition 3.1.

As described in [17], applying the Hopf construction to a map $f: S^{2 n-1} \times P^{2 n}(2) \rightarrow$ $V_{2 n+1,2}$ as in (d) yields a map $H(f): P^{4 n}(2) \rightarrow \Sigma V_{2 n+1,2}$ with $S q^{2 n}$ acting nontrivially on $H^{2 n}\left(C_{H(f)}\right)$. Since $\Sigma^{2} V_{2 n+1,2} \simeq P^{2 n+2}(2) \vee S^{4 n+1}$, composing the suspension of the Hopf construction $H(f)$ with a retract $\Sigma^{2} V_{2 n+1,2} \rightarrow P^{2 n+2}(2)$ defines a map $g: P^{4 n+1}(2) \rightarrow P^{2 n+2}(2)$ with $S q^{2 n}$ acting nontrivially on $H^{2 n+1}\left(C_{g}\right)$, so (d) implies (c).

By the proof of [17, Theorem 3.1], (c) implies (e), and (e) implies (f). The triviality of the Whitehead product $\left[i_{2 n-1}^{\prime}, j_{2 n}^{\prime}\right] \in \pi_{4 n-2}\left(V_{2 n+1,2} ; \mathbb{Z} / 2 \mathbb{Z}\right)$ when $n=1,2$ or 4 is implied by [17, Theorem 2.1], for example, and Proposition 3.1 implies $\left[i_{15}^{\prime}, j_{16}^{\prime}\right] \in$ $\pi_{30}\left(V_{17,2} ; \mathbb{Z} / 2 \mathbb{Z}\right)$ is trivial as well since $\left[i_{16}, i_{16}\right] \in \pi_{31}\left(P^{17}(2)\right)$ is divisible by 2 by $[\mathbf{1 0}$, Lemma 3.10]. Thus (f) implies (d). 


\section{A loop space decomposition of $J_{3}\left(S^{2}\right)$}

In this section, we consider some relations between the fibre bundle $S^{4 n-1} \rightarrow$ $V_{4 n+1,2} \rightarrow S^{4 n}$ defined by projection onto the first vector of an orthonormal 2-frame in $\mathbb{R}^{4 n+1}$ (equivalently, the unit tangent bundle over $S^{4 n}$ ) and the fibration $B W_{n} \rightarrow$ $\Omega^{2} S^{4 n+1}\{2\} \rightarrow W_{2 n}$ of Lemma 2.2. Letting $\partial: \Omega S^{4 n} \rightarrow S^{4 n-1}$ denote the connecting map of the first fibration, we will show that there is a morphism of homotopy fibrations

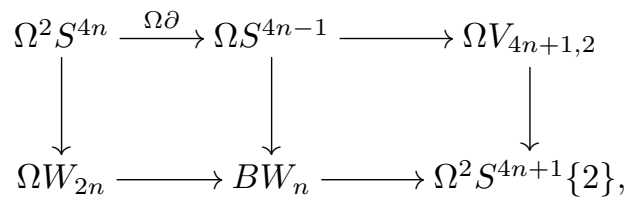

from which it will follow that for $n=1,2$ or 4 , $\Omega$ lifts through $\Omega \phi_{n}: \Omega^{3} S^{4 n+1} \rightarrow$ $\Omega S^{4 n-1}$. If this lift can be chosen to be $\Omega^{2} E$, then it follows that there is a homotopy pullback diagram

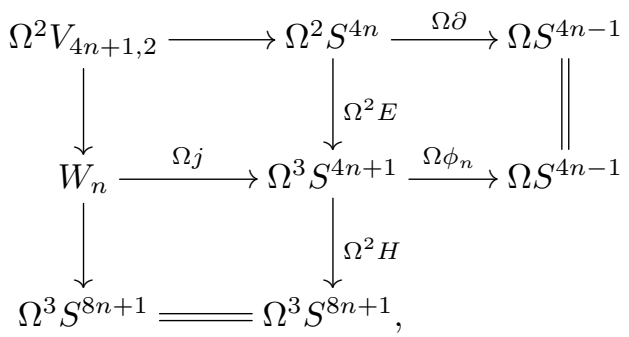

which identifies $\Omega^{2} V_{4 n+1,2}$ with $\Omega M_{3}(n)$ where $\left\{M_{k}(n)\right\}_{k \geqslant 1}$ is the filtration of $B W_{n}$ studied in [7] beginning with the familiar spaces $M_{1}(n) \simeq \Omega S^{4 n-1}$ and $M_{2}(n) \simeq$ $S^{4 n-1}\{\underline{2}\}$. (Spaces are localized at an odd prime throughout [7] but the construction of the filtration works in the same way for $p=2$.) We verify this (and deloop it) for $n=1$ since it leads to an interesting loop space decomposition which gives isomorphisms $\pi_{k}\left(V_{5,2}\right) \cong \pi_{k}\left(J_{3}\left(S^{2}\right)\right)$ for all $k \geqslant 3$.

In his factorization of the 4th-power map on $\Omega^{2} S^{2 n+1}$ through the double suspension, Theriault constructs in [18] a space $A$ and a map $\bar{E}: A \rightarrow \Omega S^{2 n+1}\{2\}$ with the following properties:

(a) $H_{*}(A) \cong \Lambda\left(x_{2 n-1}, x_{2 n}\right)$ with Bockstein $\beta x_{2 n}=x_{2 n-1}$;

(b) $\bar{E}$ induces a monomorphism in homology;

(c) There is a homotopy fibration $S^{2 n-1} \rightarrow A \rightarrow S^{2 n}$ and a homotopy fibration diagram

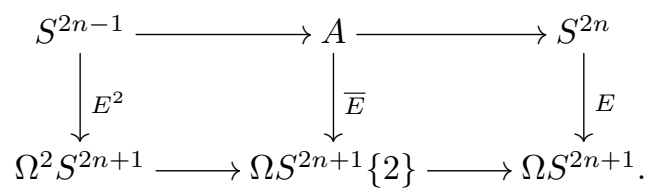

Noting that the homology of $A$ is isomorphic to the homology of the unit tangent bundle $\tau\left(S^{2 n}\right)$ as a coalgebra over the Steenrod algebra, Theriault raises the question 
of whether $A$ is homotopy equivalent to $\tau\left(S^{2 n}\right)=V_{2 n+1,2}$. Our next proposition shows this is true for any space $A$ with the properties above.

Proposition 4.1. There is a homotopy equivalence $A \simeq V_{2 n+1,2}$.

Proof. First we show that $A$ splits stably as $P^{2 n} \vee S^{4 n-1}$. As in [18], let $Y$ denote the $(4 n-1)$-skeleton of $\Omega S^{2 n+1}\{2\}$. Consider the homotopy fibration

$$
\Omega S^{2 n+1}\{2\} \longrightarrow \Omega S^{2 n+1} \stackrel{2}{\longrightarrow} \Omega S^{2 n+1}
$$

and recall that $H_{*}\left(\Omega S^{2 n+1}\{2\}\right) \cong H_{*}\left(\Omega S^{2 n+1}\right) \otimes H_{*}\left(\Omega^{2} S^{2 n+1}\right)$. Restricting the fibre inclusion to $Y$ and suspending once we obtain a homotopy commutative diagram

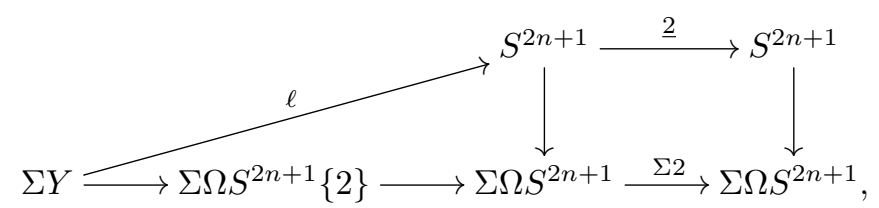

where 2 is the degree 2 map, the vertical maps are inclusions of the bottom cell of $\Sigma \Omega S^{2 n+1}$ and a lift $\ell$ inducing an isomorphism in $H_{2 n+1}()$ exists since $\Sigma Y$ is a $4 n$-dimensional complex and $\operatorname{sk}_{4 n}\left(\Sigma \Omega S^{2 n+1}\right)=S^{2 n+1}$. It follows from the James splitting $\Sigma \Omega S^{2 n+1} \simeq \bigvee_{i=1}^{\infty} S^{2 n i+1}$ and the commutativity of the diagram that $\underline{2} \circ \ell$ is nullhomotopic, so, in particular, $\Sigma \ell$ lifts to the fibre $S^{2 n+2}\{\underline{2}\}$ of the degree 2 map on $S^{2 n+2}$. Since $H_{*}\left(S^{2 n+2}\{\underline{2}\}\right) \cong \mathbb{Z} / 2 \mathbb{Z}\left[u_{2 n+1}\right] \otimes \Lambda\left(v_{2 n+2}\right)$ with $\beta v_{2 n+2}=u_{2 n+1}$, this implies $\Sigma \ell$ factors through a map $r: \Sigma^{2} Y \rightarrow P^{2 n+2}(2)$ which is an epimorphism in homology by naturality of the Bockstein, and hence $P^{2 n+2}(2)$ is a retract of $\Sigma^{2} Y$. (Alternatively, $r$ can be obtained by suspending a lift $\Sigma Y \rightarrow S^{2 n+1}\{\underline{2}\}$ of $\ell$ and using the well-known fact that $\Sigma S^{2 n+1}\{\underline{2}\}$ splits as a wedge of Moore spaces.) Now since $\bar{E}: A \rightarrow \Omega S^{2 n+1}\{2\}$ factors through $Y$ and induces a monomorphism in homology, composing $\Sigma^{2} A \rightarrow \Sigma^{2} Y$ with the retraction $r$ shows that $\Sigma^{2} A \simeq$ $\Sigma^{2}\left(P^{2 n}(2) \vee S^{4 n-1}\right)$.

Next, let $E^{\infty}: A \rightarrow Q A$ denote the stabilization map and let $F$ denote the homotopy fibre of a map $g: Q P^{2 n}(2) \rightarrow K(\mathbb{Z} / 2 \mathbb{Z}, 4 n-2)$ representing the mod-2 cohomology class $u_{2 n-1}^{2} \in H^{4 n-2}\left(Q P^{2 n}(2)\right)$. A homology calculation shows that the $(4 n-1)$ skeleton of $F$ is a three-cell complex with homology isomorphic to $\Lambda\left(x_{2 n-1}, x_{2 n}\right)$ as a coalgebra. The splitting $\Sigma^{2} A \simeq \Sigma^{2}\left(P^{2 n}(2) \vee S^{4 n-1}\right)$ gives rise to a map $\pi_{1}: Q A \simeq$ $Q P^{2 n}(2) \times Q S^{4 n-1} \rightarrow Q P^{2 n}(2)$ inducing isomorphisms in $H_{2 n-1}()$ and $H_{2 n}()$, and since the composite $g \circ \pi_{1} \circ E^{\infty}: A \rightarrow K(\mathbb{Z} / 2 \mathbb{Z}, 4 n-2)$ is nullhomotopic, there is a lift $A \rightarrow F$ inducing isomorphisms in $H_{2 n-1}()$ and $H_{2 n}($ ). The coalgebra structure of $H_{*}(A)$ then implies this lift is a $(4 n-1)$-equivalence and the result follows as $V_{2 n+1,2}$ can similarly be seen to be homotopy equivalent to the $(4 n-1)$-skeleton of $F$.

The homotopy commutative diagram (2) is now obtained by noting that the composite $\Omega S^{4 n-1} \rightarrow \Omega V_{4 n+1,2} \stackrel{\Omega \bar{E}}{\longrightarrow} \Omega^{2} S^{4 n+1}\{2\}$ is homotopic to $\Omega S^{4 n-1} \stackrel{\Omega E^{2}}{\longrightarrow}$ $\Omega^{3} S^{4 n+1} \rightarrow \Omega^{2} S^{4 n+1}\{2\}$, which in turn is homotopic to a composite $\Omega S^{4 n-1} \rightarrow B W_{n} \rightarrow \Omega^{2} S^{4 n+1}\{2\}$ since by Theorem 2.1 there is a homotopy fibration 
diagram

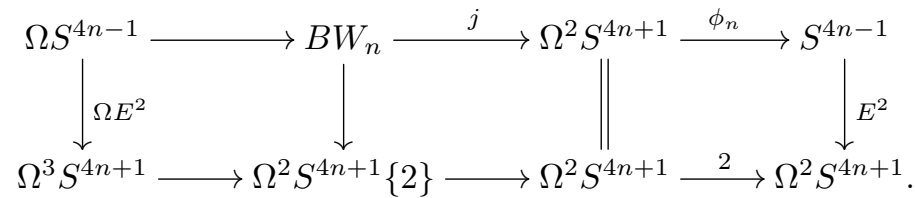

Specializing to the case $n=1$, the proof of Proposition 4.3 will show that $\Omega V_{5,2}$ fits in a delooping of diagram (3) and hence that $\Omega V_{5,2} \simeq M_{3}(1)$. We will need the following cohomological characterization of $V_{5,2}$.

Lemma 4.2. Let $E$ be the total space of a fibration $S^{3} \rightarrow E \rightarrow S^{4}$. If $E$ has integral cohomology group $H^{4}(E ; \mathbb{Z})=\mathbb{Z} / 2 \mathbb{Z}$ and mod-2 cohomology ring $H^{*}(E)$ an exterior algebra $\Lambda(u, v)$ with $|u|=3$ and $|v|=4$, then $E$ is homotopy equivalent to the Stiefel manifold $V_{5,2}$.

Proof. As shown in [22, Theorem 5.8], the top row of the homotopy pullback diagram

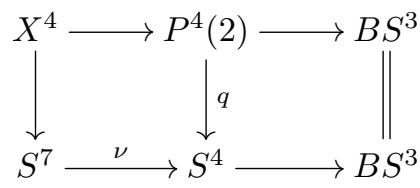

induces a split short exact sequence

$$
0 \longrightarrow \mathbb{Z} / 4 \mathbb{Z} \longrightarrow \pi_{6}\left(P^{4}(2)\right) \longrightarrow \pi_{5}\left(S^{3}\right) \longrightarrow 0,
$$

from which it follows that $\pi_{6}\left(P^{4}(2)\right)=\mathbb{Z} / 4 \mathbb{Z}\{\lambda\} \oplus \mathbb{Z} / 2 \mathbb{Z}\left\{\widetilde{\eta}_{3}^{2}\right\}$ where $\lambda$ is the attaching map of the top cell of $V_{5,2}$ and $\widetilde{\eta}_{3}^{2}$ maps to the generator $\eta_{3}^{2}$ of $\pi_{5}\left(S^{3}\right)$. It follows from the cohomological assumptions that $E \simeq P^{4}(2) \cup_{f} e^{7}$, where $f=a \lambda+b \widetilde{\eta}_{3}^{2}$ for some $a \in \mathbb{Z} / 4 \mathbb{Z}, b \in \mathbb{Z} / 2 \mathbb{Z}$, and that $H_{*}(\Omega E)$ is isomorphic to a polynomial algebra $\mathbb{Z} / 2 \mathbb{Z}\left[u_{2}, v_{3}\right]$. Since the looped inclusion $\Omega P^{4}(2) \rightarrow \Omega E$ induces the abelianization map $T\left(u_{2}, v_{3}\right) \rightarrow \mathbb{Z} / 2 \mathbb{Z}\left[u_{2}, v_{3}\right]$ in homology, it is easy to see that the adjoint $f^{\prime}: S^{5} \rightarrow \Omega P^{4}(2)$ of $f$ has Hurewicz image $\left[u_{2}, v_{3}\right]=u_{2} \otimes v_{3}+v_{3} \otimes u_{2}$ and hence $f$ is not divisible by 2. Moreover, since $E$ is an $S^{3}$-fibration over $S^{4}$, the pinch map $q: P^{4}(2) \rightarrow S^{4}$ must extend over $E$. This implies the composite $S^{6} \stackrel{f}{\rightarrow} P^{4}(2) \stackrel{q}{\rightarrow} S^{4}$ is nullhomotopic and therefore $b=0$ by the commutativity of the diagram above. It now follows that $f= \pm \lambda$ which implies $E \simeq V_{5,2}$.

Proposition 4.3. There is a homotopy fibration

$$
V_{5,2} \longrightarrow J_{3}\left(S^{2}\right) \longrightarrow K(\mathbb{Z}, 2)
$$

which is split after looping.

Proof. Let $h$ denote the composite $\Omega S^{3}\langle 3\rangle \rightarrow \Omega S^{3} \stackrel{H}{\rightarrow} \Omega S^{5}$ and consider the pullback

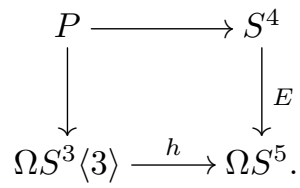


Since $h$ has homotopy fibre $S^{3}$, so does the map $P \rightarrow S^{4}$. Next, observe that $P$ is the homotopy fibre of the composite $\Omega S^{3}\langle 3\rangle \stackrel{h}{\rightarrow} \Omega S^{5} \stackrel{H}{\rightarrow} \Omega S^{9}$ and since $\Omega S^{9}$ is 7-connected, the inclusion of the 7 -skeleton of $\Omega S^{3}\langle 3\rangle$ lifts to a map $\operatorname{sk}_{7}\left(\Omega S^{3}\langle 3\rangle\right) \rightarrow P$. Recalling that $H^{4}\left(\Omega S^{3}\langle 3\rangle ; \mathbb{Z}\right) \cong \mathbb{Z} / 2 \mathbb{Z}$ and $H_{*}\left(\Omega S^{3}\langle 3\rangle\right) \cong \Lambda\left(u_{3}\right) \otimes \mathbb{Z} / 2 \mathbb{Z}\left[v_{4}\right]$ with generators in degrees $\left|u_{3}\right|=3$ and $\left|v_{4}\right|=4$, it follows that this lift must be a homology isomorphism and hence a homotopy equivalence. So $P$ is homotopy equivalent to the total space of a fibration satisfying the hypotheses of Lemma 4.2 and there is a homotopy equivalence $P \simeq V_{5,2}$.

It is well known that the iterated composite of the $p$ th James-Hopf invariant $H^{\circ k}: \Omega S^{2 n+1} \rightarrow \Omega S^{2 n p^{k}+1}$ has homotopy fibre $J_{p^{k}-1}\left(S^{2 n}\right)$, the $\left(p^{k}-1\right)$ st stage of the James construction on $S^{2 n}$. The argument above identifies $V_{5,2}$ with the homotopy fibre of the composite

$$
\Omega S^{3}\langle 3\rangle \longrightarrow \Omega S^{3} \stackrel{H}{\longrightarrow} \Omega S^{5} \stackrel{H}{\longrightarrow} \Omega S^{9},
$$

so there is a homotopy pullback diagram

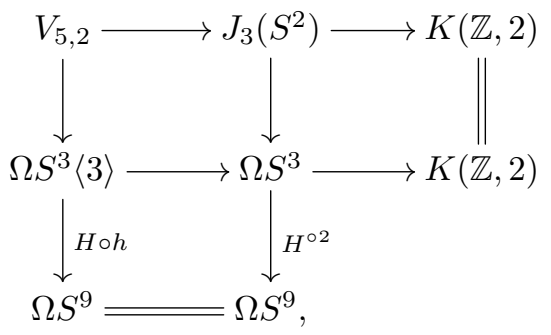

where the maps into $K(\mathbb{Z}, 2)$ represent generators of $H^{2}\left(J_{3}\left(S^{2}\right) ; \mathbb{Z}\right) \cong \mathbb{Z}$ and $H^{2}\left(\Omega S^{3} ; \mathbb{Z}\right) \cong \mathbb{Z}$. To see that the homotopy fibration along the top row splits after looping, note that the connecting map $\Omega K(\mathbb{Z}, 2)=S^{1} \rightarrow V_{5,2}$ is nullhomotopic since $V_{5,2}$ is simply-connected. Therefore the looped projection map $\Omega J_{3}\left(S^{2}\right) \rightarrow S^{1}$ has a right homotopy inverse producing a splitting $\Omega J_{3}\left(S^{2}\right) \simeq S^{1} \times \Omega V_{5,2}$.

Corollary 4.4. $\pi_{k}\left(J_{3}\left(S^{2}\right)\right) \cong \pi_{k}\left(V_{5,2}\right)$ for all $k \geqslant 3$.

\section{References}

[1] H.E.A. Campbell, F.R. Cohen, F.P. Peterson and P.S. Selick, The space of maps of Moore spaces into spheres, Proc. of John Moore Conf. on Alg. Top. and Alg. K-Theory, 72-100, Ann. of Math. Stud., 113, Princeton Univ. Press, Princeton, 1987.

[2] F.R. Cohen, A course in some aspects of classical homotopy theory, Alg. Top., Proc. Seattle 1985 (H. Miller and D. Ravenel, eds.), 1-92, Lecture Notes in Math., 1286, Springer, Berlin, 1987.

[3] F.R. Cohen, Fibration and product decompositions in nonstable homotopy theory, Handbook of Algebraic Topology, 1175-1208, North Holland, Amsterdam, 1995.

[4] F.R. Cohen, Two-primary analogues of Selick's theorem and the Kahn-Priddy theorem for the 3-sphere, Topology 23 (1984), 401-421. 
[5] F.R. Cohen and P.S. Selick, Splittings of two function spaces, Q. J. Math. 41 (1990), 145-153.

[6] B. Gray, On the iterated suspension, Topology 27 (1988), 301-310.

[7] J. Grbić, S. Theriault and H. Zhao, Properties of Selick's filtration of the double suspension $E^{2}$, J. Topol. Anal. 6 (2014), 421-440.

[8] M.A. Hill, M.J. Hopkins and D.C. Ravenel, On the nonexistence of elements of Kervaire invariant one, Ann. of Math. 184 (2016), 1-262.

[9] J. Mukai, On the attaching map in the Stiefel manifold of 2-frames, Math. J. Okayama Univ. 33 (1991), 177-188.

[10] J. Mukai and T. Shinpo, Some homotopy groups of the mod 4 Moore space, J. Fac. Sci. Shinshu Univ. 34 (1999), 1-14.

[11] J. Mukai and A. Skopenkov, A direct summand in a homotopy group of the mod 2 Moore space, Kyushu J. Math. 58 (2004), 203-209.

[12] D.C. Ravenel, The non-existence of odd primary Arf invariant elements in stable homotopy theory, Math. Proc. Cambridge Philos. Soc. 83 (1978), 429443.

[13] W. Richter, A conjecture of Gray and the $p^{\text {th }}$ power map on $\Omega^{2} S^{2 n p+1}$, Proc. Amer. Math. Soc. 142 (2014), 2151-2160.

[14] P.S. Selick, Odd primary torsion in $\pi_{k}\left(S^{3}\right)$, Topology 17 (1978), 407-412.

[15] P.S. Selick, A decomposition of $\pi_{*}\left(S^{2 p+1} ; \mathbb{Z} / p \mathbb{Z}\right)$, Topology 20 (1981), 175-177.

[16] P.S. Selick, A reformulation of the Arf invariant one mod p problem and applications to atomic spaces, Pacific J. Math. 108 (1983), 431-450.

[17] P.S. Selick, Indecomposability of the Stiefel manifolds $V_{m, 3}$, Topology 27 (1988), 479-485.

[18] S. Theriault, 2-primary Anick fibrations, J. Topol. 4 (2011), 479-503.

[19] S. Theriault, A case when the fibre of the double suspension is the double loops on Anick's space, Canad. Math. Bull. 53 (2010), 730-736.

[20] S. Theriault, A new proof of the odd primary homotopy exponent of spheres, Manuscripta Math. 139 (2012), 137-151.

[21] S. Theriault, Anick's fibration and the odd primary homotopy exponent of spheres, arXiv:0803.3205.

[22] J. Wu, Homotopy theory of the suspensions of the projective plane, Mem. Amer. Math. Soc. 162 (2003), No. 769.

Steven Amelotte steven.amelotte@mail.utoronto.ca

Department of Mathematics, University of Toronto, 40 St. George Street, Toronto, Ontario, M5S 2E4, Canada 